\title{
Essai de traitement, dans les conditions africaines, du polyparasitisme du zébu, à l'aide des associations Disto-5-Thiabendazole, Disto-5-Tartrate de Morantel et Disto-5-Tétramisole
}

\author{
par M. GRABER $(*)$, P. TAGER-KAGAN $(* *)$, R. DELAVENAY $(* * *)$ \\ et C. PERROTIN (*) \\ (avec la collaboration technique de MM. DJIBO GERBA et GEBRE NEGUS TESFAMARIAN)
}

\begin{abstract}
RÉSUMÉ
Les auteurs exposent les résultats positifs qu'ils ont obtenus en Afrique dans la lutte contre le polyparasitisme du zébu, à base de nématodes et trématodes, grâce à l'emploi d'associations médicamenteuses présentant un large spectre d'activité dont ils donnent la composition et la formulation. Ils insistent sur la nécessité de n'utiliser en Afrique, que des associations sous marque déposée, fabriquées par des firmes garantissant rigoureusement leur production, présentées sous forme de comprimés séccables exactement dosés.
\end{abstract}

Parmi les nombreuses affections dont souffre le bétail en Afrique tropicale, les maladies parasitaires tiennent une place importante en raison de leurs incidences économiques, directes ou indirectes. Celles-ci se manifestent surtout en fin de saison sèche, lorsque les possibilités alimentaires fortement réduites aboutissent à un état de sous-alimentation, voire de sub-disette.

La conjonction de ces 2 facteurs - sousalimentation et parasitisme - se traduit par une mortalité élevée chez les jeunes et par des pertes de poids importantes (de 10 à 25 p. 100) chez les animaux de boucherie.

Les helminthiases sont dues, dans leur grande majorité à la présence, dans le tractus digestif, de 2 groupes de parasites :

(*) Chaire de parasitologie, Ecole Nationale Vétérinaire, Marcy l'Etoile, 69260 Charbonnères les Bains (France).

(**) Laboratoire de l'Elevage, B. P. 485, Niamey, République du Niger.

$(* * *)$ Mission Vétérinaire Française en Ethiopie, P. O. Box 1053, Addis-Abéba.

I. E. M. V. T., 10 rue Pierre-Curie, 94700 MaisonsAlfort (France).
- Des nématodes: Ascaris, Strongyloides, «Strongles》 (Haemonchus, Cooperia, Bunostomum, Oesophagostomum, plus rarement Trichostrongylus et Impalaia;

- Des trématodes : Paramphistomatidae du duodénum et de la panse; Fasciola et Dicrocoelium du parenchyme hépatique et des canaux biliaires: Schistosoma des veines hépatıques et mésentériques.

Les premiers se rencontrent principalement chez les jeunes zébus et les seconds chez les animaux plus âgés.

Trématodes et nématodes sont très souvent associés chez le même animal.

La fréquence du polyparasitisme rend la prophylaxie difficile. De plus en plus, on recommande (9) des traitements collectifs périodiques à but préventif, ce qui nécessite l'emploi de médicaments polyvalents à large spectre qui englobent les parasites les plus pathogènes et les plus communs des bovins d'Afrique tropicale. Ce sont : 
- Soit des composés simples comme :

- le Cambendazole (*) qui détruit en une seule prise un grand nombre de nématodes gastro-intestinaux et pulmonaires, ainsi que certains cestodes de l'intestin (16). Il est sans action sur les trématodes.

- L'Albendazole (**). Vers $10 \mathrm{mg} / \mathrm{kg}$, il permet l'élimination des nématodes gastrointestinaux et pulmonaires, de certains cestodes de l'intestin et des trématodes du foie (Fasciola). Anthelminthique récent (23), ses indications demandent à être mieux précisées en milieu tropical.

- Soit des associations médicamenteuses dont il existe de nombreuses variantes. Les plus connues sont représentées par :

- Le Ranizole ou Duofas (*) qui renferme 2,5 p. 100 de Rafoxanide et 14,67 p. 100 de Thiabendazole. Chez les bovins, la dose est de $45 \mathrm{ml}$ par $100 \mathrm{~kg}$ de poids vif, soit $66 \mathrm{mg} / \mathrm{kg}$ de Thiabendazole et $11,25 \mathrm{mg} / \mathrm{kg}$ de Rafoxanide. Compte tenu des observations faites dans divers pays d'Afrique, la dose de Rafoxanide est suffisante pour tuer les fascioles (Fasciola gigantica) adultes et celles âgées de 8 semaines (25). Elle est inactive sur les paramphistomes de la panse.

En revanche, la dose de Thiabendazole est insuffisante, car il reste encore trop d'œsophagostomes et de bunostomes immatures. II faut au moins, $100 \mathrm{mg} / \mathrm{kg}$ (7).

- Le Nilsan ou Iména(***), a ssociation à base de Tétramisole $(3,4$ p. 100$)$ et d'Oxyclozanide (3,4 p. 100). La dose, pour un bovin de moins de $300 \mathrm{~kg}$, est de 40 à $45 \mathrm{ml}$ par $100 \mathrm{~kg}$, soit $15 \mathrm{mg} / \mathrm{kg}$ d'Oxyclozanide et $15 \mathrm{mg} / \mathrm{kg}$ de Tétramisole. Cette dose est très efficace à l'égard des « Strongles » gastro-intestinaux et pulmonaires, des Fasciola gigantica de plus de 70 jours et des paramphistomes adultes (14).

L'abattage des animaux traités ne peut avoir lieu que 14 jours après l'intervention, de façon à ce que les viandes ne renferment aucun résidu pouvant être dangereux pour la santé de ceux qui les consomment : c'est le temps d'attente.

Depuis, d'autres formules ont vu le jour mettant en jeu du Bis(2-hydroxy-3,5-dichlorophényl) sulfoxyde-Bitin-S ou Bithionol sulfoxyde sous forme de Disto-5 (****) en association soit

(*) Merck Sharp and Dohme.

(**) Smith Kline and French.

(***) I. C. I.

$(* * *)$ ) Cogla S. A.

$(* * * *)$ Pfizer International. avec du Thiabendazole (*) soit avec du Tartrate de Morantel $(* * * * *)$, soit avec du Tétramisole $(* * * * * *)$.

Deux essais ont été effectués, le premier au Niger en juillet 1974 et, le second, en Ethiopie, en décembre 1976. Les résultats obtenus - fort intéressants - méritent de retenir l'attention.

\section{MATÉRIEL ET MÉTHODE}

\section{Les animaux d'expérience}

1.1. Quatre-vingt dix-neuf zébus adultes ont été utilisés dont 75 au Niger et 24 en Ethiopie $(* * * * * * * *)$. Les premiers ont été achetés sur les marchés du fleuve Niger, d'Ayorou à Say, et les seconds dans la région de Kofélé (11), Province de l'Arussi (Ethiopie).

Les zébus nigériens (61 mâles et 14 femelles) pesaient entre 83 et $256 \mathrm{~kg}$. Ils se trouvaient dans un état de maigreur extrême (Photo $n^{\circ} 1$ ) imputable aux mauvaises conditions alimentaires résultant de la sécheresse exceptionnelle qui sévissait alors au Niger, comme dans toutes les zones sahéliennes d'Afrique.

Les zébus abyssins comprenaient 24 mâles de 145 à $310 \mathrm{~kg}$. Leur état d'entretien était, dans l'ensemble, assez bon (Photo $n^{\circ} 2$ ), la saison des pluies 1976 ayant, dans le Sidamo, entraîné une repousse satisfaisante des pâturages.

1.2. Ils hébergeaient un grand nombre de parasites. Sur coprocultures, dans les fèces après traitement et à l'autopsie, ont été identifiés :

- Dicrocoelium hospes, canaux biliaires, $\mathrm{Ni}$ ger : 4 animaux.

- Fasciola gigantica, canaux biliaires : 56 animaux dont 47 au Niger et 9 en Ethiopie.

- Fasciola hepatica, canaux biliaires, Ethiopie : 20 animaux $(* * * * * * *)$.

- Paramphistomatidae, panse :

- Stephanopharynx compactus, Niger : un animal.

- Paramphistomum clavula

- Paramphistomum phillerouxi

23 animaux (22).

(******) Rhône-Poulenc.

(*******) Plus un essai complémentaire portant sur 13 zébus ayant reçu $60 \mathrm{mg} / \mathrm{kg}$ de Bitin-S.

$(* * * * * * * *)$ En Ethiopie, 5 animaux hébergeaient à la fois Fasciola hepatica et Fasciola gigantica. 
- Paramphistomum daubneyi, Ethiopie : 24 animaux.

- Carmyerius spatiosus : 7 animaux, Niger (22).

- Schistosoma bovis, veines mésentériques, Niger : 42 animaux.

- Echinococcus polymorphus, foie et poumon, Ethiopie : 17 animaux.

— «Strongles » gastro-intestinaux :

- Oesophagostomum (Bosicola) radiatum, gros intestin et cacum : 72 animaux dont 67 au Niger et 5 en Ethiopie.

- Bunostomum phlebotomum, duodénum : 7 animaux dont 6 au Niger et 1 en Ethiopie.

- Trichostrongylus sp., intestin, Ethiopie : un animal.

- Cooperia punctata / duodénum : 74 animaux

- Cooperia dont 69 au Niger et 5 en pectinata Ethiopie.

- Haemonchus contortus, caillette : 84 animaux dont 73 au Niger et 11 en Ethiopie.

- Onchocerca armillata, aorte, Niger : 17 animaux.

- Setaria labiato-papillosa, péritoine, Niger : 20 animaux.

- Parafilaria bosicola, conjonctif sous-cutané, Niger : 1 animal.

- Gongylonema pulchrum, œsophage, Ethiopie : 1 animal.

- Thelazia rhodesii, ail : 39 animaux dont 36 au Niger et 3 en Ethiopie.

Dans la totalité des cas, trématodes et nématodes étaient associés entre eux, le type d'association le plus couramment rencontré étant à base de 3 ou de 4 helminthes différents ( 2 trématodes et 1 ou 2 nématodes).

1.3. Les animaux ont été placés sous des abris provisoires non fermés et couverts, de manière à les protéger du soleil et de la pluie. Ils sont isolés un par un. Le sol est bétonné (Ethiopie) ou compacté à la latérite (Niger), ce qui permet de recueillir régulièrement les matières fécales évacuées.

La nourriture est constituée de paille de riz sèche (Niger) ou de tiges et de feuilles de maïs (Ethiopie). L'abreuvement se fait au seau.

En Ethiopie, la présence de nombreuses hyènes rend, durant la nuit, le gardiennage difficile.
Aussi les animaux, après traitement, ont-ils été maintenus en stalle séparée juste le temps nécessaire à l'expulsion des nématodes ( 72 heures). Ils sont ensuite remis dans le troupeau.

\section{Les associations médicamenteuses}

Les anthelminthiques entrant dans la composition des associations ont déjà été étudiés en Afrique tropicale et leur posologie est connue ( 7 , $8,10,13)$. Quatre types d'associations ont été expérimentés au Niger :

- le Wormyl 10, en boîtes de 100 comprimés de $20 \mathrm{~g}$. Chaque comprimé renferme $4 \mathrm{~g}$ de Disto-5 et $10 \mathrm{~g}$ de Thiabendazole $(100 \mathrm{mg} / \mathrm{kg})$. On administre par la voie buccale un comprimé par $100 \mathrm{~kg}$ de poids vif;

- Le Wormyl 8. La présentation est la même. La quantité de Thiabendazole incorporée à chaque comprimé n'est plus que de $8 \mathrm{~g}$, soit $80 \mathrm{mg} / \mathrm{kg}$. On administre, également par la voie buccale, un comprimé par $100 \mathrm{~kg}$ de poids vif ;

- Du Disto-5 $(40 \mathrm{mg} / \mathrm{kg})+$ du Tartrate de Morantel à $7,5 \mathrm{mg} / \mathrm{kg}$. Administration séparée - toujours par la bouche -... de comprimés de Disto- 5 dosés à $2 \mathrm{~g}$ (un comprimé par $50 \mathrm{~kg}$ de poids vif) et de comprimés de Tartrate de Morantel dosé à $750 \mathrm{mg}\left({ }^{*}\right)$ (un comprimé pour $100 \mathrm{~kg}$ de poids vif) ;

_- Du Disto-5 $(40 \mathrm{mg} / \mathrm{kg})$ dans les mêmes conditions que précédemment et du Tartrate de Morantel à $5 \mathrm{mg} / \mathrm{kg}$, sous forme de comprimés dosés à $500 \mathrm{mg}(* *)$, soit un comprimé pour $100 \mathrm{~kg}$ de poids vif.

En Ethiopie, l'association est à base de Bithionol Sulfoxyde $(40 \mathrm{mg} / \mathrm{kg})$ et de Tétramisole (10 $\mathrm{mg} / \mathrm{kg})\left({ }^{* *}\right)$, sous forme de comprimés séccables renfermant $8 \mathrm{~g}$ de Bitin-S et $2 \mathrm{~g}$ de Tétramisole. La dose recommandée est de un comprimé pour $200 \mathrm{~kg}$ de poids vif.

\section{Méthode}

Elle associe 2 techniques différentes, celle du "Critical test » (dénombrement des vers éliminés à l'issue du traitement et comparaison, à l'examen nécropsique, avec la population survivante) et celle du "Controlled test " (comparaison, à l'autopsie, entre les populations de vers existant dans les lots d'animaux traités et dans le lot témoin).

$\left.{ }^{*}\right)=$ Morantel 750 .

$(* *)=$ Morantel 500 .

$(* * *)$ Wormex Cogla. Origine du Tétramisole: Société Rhône-Poulenc. 
Elle a été exposée en détail à plusieurs reprises pour des essais similaires $(2,7,8)$; il est donc inutile d'y revenir.

Toutefois, lors de la formation des lots, il faut tenir compte non seulement de l'âge, du poids, de la conformation et de l'état général des animaux, mais aussi du niveau de l'infestation parasitaire. Pour ce faire, on possède quelques points de repère. Chez, les bovins, on sait (6) que les infestations parasitaires deviennent dangereuses, avec passage au stade de parasitisme maladie :

- Pour Fasciola hepatica, au-delà de 200 à 300 œufs au gramme de matière fécale (pour Fasciola gigantica, de 180 à 200);

- Pour les paramphistomes, vers 3 à 4000 œufs au gramme de matière fécale ;

- Pour les Haemonchus et pour les Cooperia, vers 500 et 5000 oufs au gramme de matière fécale.

Il s'agit lả de mono-ịnfestations. Lorsque l'on a affaire à des associations parasitaires à base de Fasciola, de paramphistomes et de "Strongles » gastro-intestinaux, il importe d'établir de nouvelles moyennes.

Compte tenu des observations faites en divers points d'Afrique tropicale, le niveau de l'infestation (tous parasites réunis) peut être considéré comme :

- Faible : moins de 400 cufs au gramme de matière fécale ;

— Moyen : de 400 à 1000 cufs au gramme de matière fécale ;

— Fort : 1100 à 2500 œufs au gramme de matière fécale ;

— Massif : au-delà.

Ont été constitués :

- Au Niger, 5 lots de 15 animaux (un lot témoin et un lot pour chaque association médicamenteuse) comprenant chacun un tiers de zébus faiblement parasités, un tiers de zébus moyennement parasités et un tiers de zébus massivement parasités ;

- En Ethiopie, 2 lots : un lot témoin de 10 zébus et un lot traité au Wormex (14 animaux).

\section{RÉSULTATS}

\section{TRÉMATODES}

\subsection{Dicrocoelium hospes}

Les associations Disto-5 + Morantel 500, Disto-5 + Morantel 750 et Wormyl 8 sont inactives et, à l'autopsie, on retrouve, dans les canaux biliaires et dans la vésicule, plusieurs centaines, voire plusieurs milliers de petites douves bien vivantes.

\subsection{Schistosoma bovis}

Les résultats sont identiques. Les associations sont parfaitement inefficaces. La comparaison du niveau de l'infestation bilharzienne chez les animaux traités et chez les témoins n'indique aucune diminution du nombre de trématodes chez les zébus ayant reçu les associations médicamenteuses.

\subsection{Fasciola hepatica et Fasciola gigantica}

Le Bitin-S à $40 \mathrm{mg} / \mathrm{kg}$ est très actif sur Fasciola hepatica et Fasciola gigantica adultes, ainsi que sur les fascioles un peu plus jeunes de 75-90 jours n'ayant pas encore atteint leur complète maturité sexuelle (*).

Sur les 76 animaux traités, il ne restait qu'un seul animal porteur de parasites: il s'agissait d'un zébu éthiopien atteint d'une infestation mixte par Fasciola hepatica et par Fasciola gigantica. Quelques Fasciola hepatica, vivantes et de petite taille, ont été isolées dans l'un des canaux biliaires. Celui-ci, épaissi et très dilaté, formait une véritable poche à l'intérieur du foie (voir infra).

Le pourcentage d'efficacité sur les douves âgées de plus de 8 semaines se situe donc autour de 99 p. 100, ce qui confirme les observations antérieures $(10,13)$.

\subsection{Paramphistomatidés}

Au Niger, 72 p. 100 des zébus traités sont totalement débarrassés de leurs parasites.' En fait, ce pourcentage cache 2 réalités différentes:

- Le médicament est très efficace à l'égard des Paramphistomum et des Carmyerius sexuellement mûrs. Après examens coproscopiques, 35 des 60 animaux ayant reçu du Bitin-S ont été reconnus porteurs de trématodes gastriques en état de pondre. Le jour de l'autopsie, il n'existait plus aucun parasite adulte dans la panse, alors que, sur l'ensemble des témoins, ont été recueillis 370 Carmyerius spatiosus et $7710 \mathrm{~Pa}$ -

(*) Les critères permettant d'apprécier l'âge de Fasciola gigantica sont basés sur la longueur des parasites obtenus après infestations de jeunes zébus autopsiés à intervalles réguliers (18). 
ramphistomum clavula et Paramphistomum phillerouxi, soit, en moyenne, 538 vers par animal.

On sait que les paramphistomatidés, après avoir séjourné durant 5 à 7 semaines dans le duodénum, effectuent une migration rétrograde qui les mène dans le rumen où ils acquièrent leur maturité sexuelle. Tous les parasites ne gagnent pas ensemble cet organe. Il se produit souvent un certain décalage dans le temps, surtout en cas d'infestation massive. Aussi, observe-t-on fréquemment dans la panse un mélange de paramphistomes à divers stades d'évolution.

Les trématodes encore immatures sont beaucoup moins sensibles à l'action du Disto-5. Un certain nombre d'entre eux résistent à l'action du médicament, soit, dans le cas de cette expérience, 42 Carmyerius spatiosus et 131 Paramphistomum clavula et Paramphistomum phillerouxi. Le nombre de parasites dénombrés à l'autopsie varie de 1 à 47 individus par animal. Dans l'ensemble, il demeure faible : moins de 10 trématodes dans 60 p. 100 des cas.
En Ethiopie, 50 p. 100 des zébus parasités (7 sur 14) sont, après traitement au Bithionol Sulfoxyde, encore porteurs de Paramphistomum daubneyi. Comme dans le cas précédent, le médicament agit surtout sur les Trématodes adultes. A l'autopsie du lot "Wormex", 1379 parasites, au total, ont été comptés contre 20490 chez les témoins, ce qui représente un pourcentage d'efficacité de 93 p. 100 . Il ne reste chez les animaux traités qu'un petit nombre de paramphistomes adultes : $825,2,151,74,3,4$ contre $356,8902,3575,2956,444,1912,20$, 1484,193 et 148 chez les témoins.

Un seul animal hébergeait des trématodes mûrs (320) et des trématodes immatures (960) associés.

Les résultats obtenus sont donc beaucoup moins favorables qu'au Niger ou au Tchad (13).

\section{Nématodes}

Les résultats figurent aux tableaux 1 et 2 .

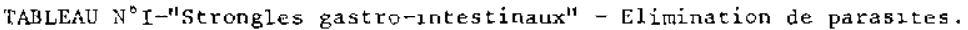

\begin{tabular}{|c|c|c|c|c|}
\hline Associations & \multicolumn{4}{|c|}{ Nombre total de parasites expulsês } \\
\hline & 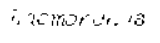 & 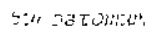 & inderengustomer & CLipenis \\
\hline Morante $1750+$ Insto-5 & $22::$ & - & 266 & 13 \\
\hline Morantel $500+$ Disto-5 & 10 & - & 72 & 1 \\
\hline Wormy 18 & 2 & - & 27 & - \\
\hline Wormyl 10 & 32 & - & $162::$ & $1:: 2::$ \\
\hline Wormex & - & 10 & 25 & - \\
\hline
\end{tabular}

$\because$ dont un adulte immature ; $::$ dont une larve $\mathrm{L}_{5} ; \because: \because$ adulte immature.

TABLEAU N*II-Nombre total de Nêmatodes gastro-intestinaux rencontrés à l'autopsie.

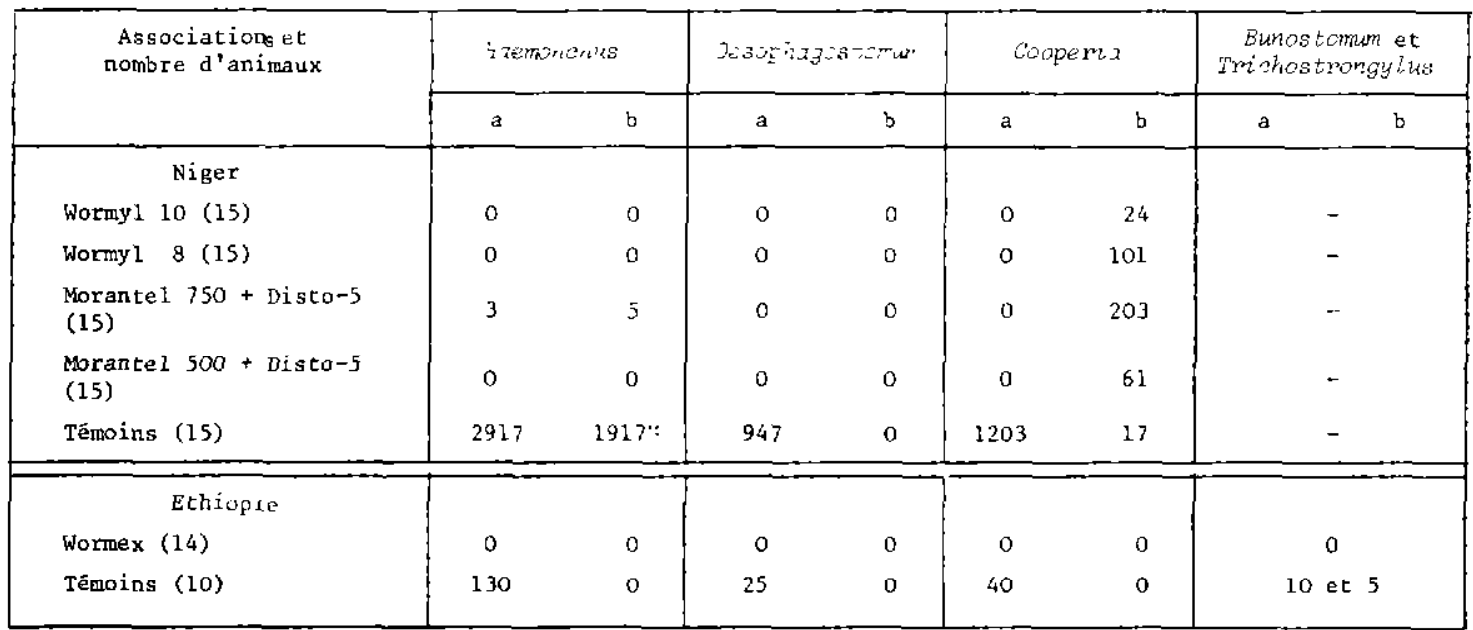

$a=$ adultes et mûrs $; b=$ immatures $: L_{4}, L_{5}$ et adultes immatures ;

$\because$ femelles adultes mûres et immatures mêlangées. 
2.1. Au Niger ou en Ethiopie, que ce soit avec le Thiabendazole avec le Morantel ou avec le Tétramisole, les cesophagostomes adultes et mûrs sont, aux doses utilisées, expulsés dans leur totalité. Le caecum et le gros intestin sont vides de parasites. Les larves $\mathbf{L}_{5}$ libres dans l'intestin paraissent également sensibles à l'action du Thiabendazole.

2.2 Les Haemonchus adultes, mûrs ou immatures, sont également chassés par le Thiabendazole à 80 et $100 \mathrm{mg} / \mathrm{kg}$, ainsi que par le Tétramisole (*). Avec le Morantel, les résultats sont paradoxaux. Il ne reste plus d'Haemonchus à $5 \mathrm{mg} / \mathrm{kg}$, mais, à $7,5 \mathrm{mg} / \mathrm{kg}, 2$ animaux sont encore parasités, le premier par 3 nématodes adultes et le second par 5 vers adultes et immatures. Le pourcentage d'efficacité est de 99,8 p. 100. Au Tchad, c'est l'inverse qui se produit : il demeure, dans la caillette et dans l'intestin, quelques Haemonchus et quelques osophagostomes à $5 \mathrm{mg} / \mathrm{kg}$, tandis qu'à $7,5 \mathrm{mg} / \mathrm{kg}$, les nématodes cessent d'exister.

Les doses de 5 et de $7,5 \mathrm{mg} / \mathrm{kg}$ semblent donc être des doses limites qui laissent subsister quelques parasites.

2.3. Soixante-quatorze animaux, au Niger, hébergeaicnt des Cooperia gravides dont les œufs en coproculture donnent des larves $\mathbf{L}_{3}$.

Le Thiabendazole à 80 et $100 \mathrm{mg} / \mathrm{kg}$, le Morantel à 5 et $7,5 \mathrm{mg} / \mathrm{kg}$, le Tétramisole à $10 \mathrm{mg} / \mathrm{kg}$ détruisent tous les Nématodes et, le jour de l'autopsie, les Cooperia appartenant à cette classe d'âge ont tous disparu (tabl. II).

Cependant, - toujours au Niger - 34 zébus se sont révélés porteurs de Cooperia immatures (12) provenant d'infestations acquises au cours de la saison des pluies précédente, les parasites demeurant toute la saison sèche en état d'hypobiose sous forme de larves $\mathrm{L}_{4}$ intrapariétales. Les traitements au Thiabendazole et au Morantel ne sont pas entièrement satisfaisants : à l'autopsie, on note la présence de quelques immatures - $\mathbf{L}_{4}, L_{5}$ et adultes - dont le nombre, pour chaque lot, est donné au tableau II.

Il est bien difficile d'établir avec précision l'efficacité de ces deux anthelminthiques à l'égard des larves de Cooperia inhibées. Dans le cas présent, il n'est pas possible de comparer le

(*) Après le traitement, les Haemonchus - comme les Cooperia meurent et sont digérés au cours de leur transit dans l'intestin. Quelques-uns seulement parviennent intacts à l'extérieur. niveau d'infestation des animaux traités et celui des animaux témoins. En outre, le nombre de Cooperia immatures recueillis dans l'intestin varie considérablement d'un animal à l'autre et il serait hasardeux, à partir de ces données. de tirer des conclusions définitives. Néanmoins, la lecture de divers travaux bibliographiques donne quelques indications. Pour le Thiabendazole, sur des larves (Cooperia punctata et Cooperia pectinata) de 7 jours - ce qui correspond au stade de larves $\mathrm{L}_{4}-$, le pourcentage d'efficacité diffère selon les auteurs: 93 à 97 p. 100 à $55 \mathrm{mg} / \mathrm{kg}(15), 85$ p. 100 à $100 \mathrm{mg} / \mathrm{kg}$ et $93 \mathrm{p} .100$ à $103-117 \mathrm{mg} / \mathrm{kg}$ (1).Indéniablement, le Thiabendazole détruit un certain nombre de larves $\mathrm{L}_{4}$, mais, même à des doses supérieures à $100 \mathrm{mg} / \mathrm{kg}$, il ne faut pas compter les faire disparaître complètement.

En ce qui concerne le Morantel, à $7,5 \mathrm{mg} / \mathrm{kg}$, l'action sur les larves $\mathrm{L}_{5}$ de Cooperia est supérieure à 90 p. $100(19)$, tandis qu'à $10 \mathrm{mg} / \mathrm{kg}$, sur les larves $\mathrm{L}_{4}$, elle paraît beaucoup moins bonne (3).

2.4. Les thélazies ont été recherchées systématiquement à l'autopsie des animaux traités et des animaux témoins.

En comparant la moyenne du nombre de nématodes recueillis chez les animaux traités et chez les témoins, on peut penser, à première vue, que les trois médicaments expérimentés sont susceptibles d'éliminer 30 à 60 p. 100 des parasites de l'oil.

En réalité, le nombre d'animaux porteurs de nématodes, ainsi que le niveau de l'infestation sont différents dans chaque lot et il est impossible de conclure valablement.

\section{COMMENTAIRES}

\section{Sur les nématodes gastro-intestinaux, adultes}

1.1. La dose de $10 \mathrm{mg} / \mathrm{kg}$ de Tétramisole semble, en Afrique tropicale, suffisante en l'absence de certains trichostrongylidés, notamment les Ostertagia et les Nematodirus qui nécessitent des posologies plus élevées.

1.2. La dose de $100 \mathrm{mg} / \mathrm{kg}$ de Thiabendazole donne des résultats satisfaisants. Chez le zébu, des essais faits sur le terrain dans le nord de la Nigeria (20) montrent qu'à cette posologie, le nombre d'aufs (Cooperia surtout) au gramme de matière fécale diminue de façon significative en fin de saison sèche. 
1.3. La dose de Morantel demande à être augmentée $(10 \mathrm{mg} / \mathrm{kg})$, car, aux doses utilisées (5 et $7,5 \mathrm{mg} / \mathrm{kg}$ ), il reste encore dans la caillette quelques Haemonchus qui, lors d'infestation massive, risquent de survivre en plus grand nombre.

2. Les formes immatures (Larves $\mathbf{L}_{4}$ ) de Cooperia punctata et de Cooperia pectinata sont, aux posologies indiquées, beaucoup plus résistantes à l'action du Thiabendazole, du Morantel et du Tétramisole $\left({ }^{*}\right)$. Celles qui, après traitement, demeurent vivantes dans l'intestin sont peu nombreuses et ne représentent pas un danger pour la santé de l'animal.

Toutefois, en fin de saison sèche, l'état d'hypobiose cesse. Les nématodes deviennent adultes et éliminent des œufs dans le milieu extérieur. $\mathrm{Si}$ les conditions clımatiques (humidité, chaleur) sont favorables, ils évoluent et donnent naissance à des larves infestantes $\mathbf{L}_{3}$ qui ensemencent les pâtúrages avec possibilité d'infestation ou de réinfestation des jeunes et des animaux plus âgés. Pour chasser les formes immatures de Cooperia, la première solution consiste à augmenter les doses de médicaments. Il n'est pas certain qu'elles se montrent plus efficaces. De plus, avec certaines associations, des troubles d'intolérance peuvent se manifester (voir infra).

Aussi, dans les zones tropicales situées au nord de l'Equateur, recommande-t-on de procéder à 2 séries de traitements dans l'année (12) :

- Le premier de septembre à novembre, selon les latitudes. Il est destiné à réduire les populations de vers acquises au cours de la saison des pluies et de toucher un certain nombre de larves déjà en état d'hypobiose ;

- Le second de mars à juin, en fin de saison sèche. Il a pour but d'achever, dans toute la mesure du possible, l'élimination des trichostrongylidés ayant survécu au traitement précédent.

\section{Disto-5}

3.1. Comme il a été dit plus haut, l'efficacité du Disto-5 $(40 \mathrm{mg} / \mathrm{kg})$ à l'égard des douves hépatiques de plus de 8 semaines est de 99 p. 100. Néanmoins, dans certaines circonstances, le médicarment fait preuve d'irrégularité (10) :

- Lorsqu'il s'agit de Fasciola hepatica plus difficile à détruire que Fasciola gigantica;

'- Lors d'infestations massives, quand il se

(*) A $10 \mathrm{mg} / \mathrm{kg}$, le pourcentage de réduction est d'environ 85 p. 100 . forme sur le trajet des plus gros canaux biliaires une ou plusieurs poches volumineuses, à parois épaisses où s'accumule un nombre plus ou moins élevé de trématodes, généralement de petite taille (11). La circulation de la bile se fait mal. Dans la lésion, la concentration de Bitin-S devient insuffisante et quelques parasites échappent à l'action du médicament.

Pour parer à cet inconvénient, il est nécessaire d'augmenter la dose et de la porter à $60 \mathrm{mg} / \mathrm{kg}$. En effet, un essai complémentaire effectué en Ethiopie sur 13 zébus atteints de distomatose massive (à Fasciola hepatica surtout) a montré qu'à cette dose, les douves enfermées dans les poches hépatiques étaient tuées, en totalité par l'anthelminthique.

En outre, cette dose a l'avantage d'agir sur les fascioles de 42 jours en cours de migration dans le parenchyme hépatique comme l'indiquent des observations faites au Tchad avec Fasciola gigantica (10). C'est d'ailleurs la dose préconisée par les auteurs russes $(5,17)\left({ }^{*}\right)$. Sur le terrain, quelques essais ont été réalisés dans le nord de la Nigeria (20) sur des zébus atteints de distomatose chronique à Fasciola gigantica. Le Bitin-S, à la dose de $40 \mathrm{mg} / \mathrm{kg}$, a été administré un mois avant (15 mars) et un mois après (15 mai) le début de la saison des pluies. Les résultats (augmentation de poids ; diminution du nombre d'œufs dans les matières fécales) sont beaucoup plus favorables dans le second que dans le premier cas.

Le traitement de la mi-mars, semble, au dosage utilisé, insuffisant, car il intervient à une époque où les douves issues des infestations de saison sèche sont encore immatures, donc moins sensibles à l'action du Disto-5. Dans les zones sahéliennes (9), il permet cependant de surmonter les effets souvent dramatiques d'une alimentation défectueuse liée à l'amenuisement des ressources fourragères à cette période de l'année.

Le traitement de la mi-mai concerne des douves beaucoup plus âgées que le médicament élimine facilement. Les ressources fourragères, du fait de la repousse des pâturages, s'améliorent et le traitement au Disto-5 s'accompagne d'une reprise de poids importante.

Dans bien des régions d'Afrique tropicale (**),

(*) Le bis (2-Hydroxy-3,5-Dichlorophényl) sulfoxyde fabriqué en U. R.S. S. est connu sous le nom de Sulphène.

$(* *)$ En Ethiopie, les infestations par Fasciola hepa. tica, du fait de la biologie propre du secteur (Lymnaea truncatula), se manifestent surtout à la fin de la saison des pluies (octobre-décembre). 
le problème de la distomatose bovine ne peut être dissocié du problème alimentaire qui se pose, en général, à la fin de la saison sèche. Pour tenter d'améliorer la situation, on ne peut agir, dans l'état actuel de nos connaissances, que sur le parasite. Comme, la plupart du temps, les fascioles sont encore immatures, il faut dis- tribuer des doses susceptibles d'en supprimer le plus grand nombre et ce résultat ne peut être obtenu qu'à la posologie de $60 \mathrm{mg} / \mathrm{kg}$.

3.2. Sur les paramphistomes de la panse, il est souhaitable d'utiliser la même dose. Sur les 13 animaux (essai complémentaire) traités en

LES ANIMAUX

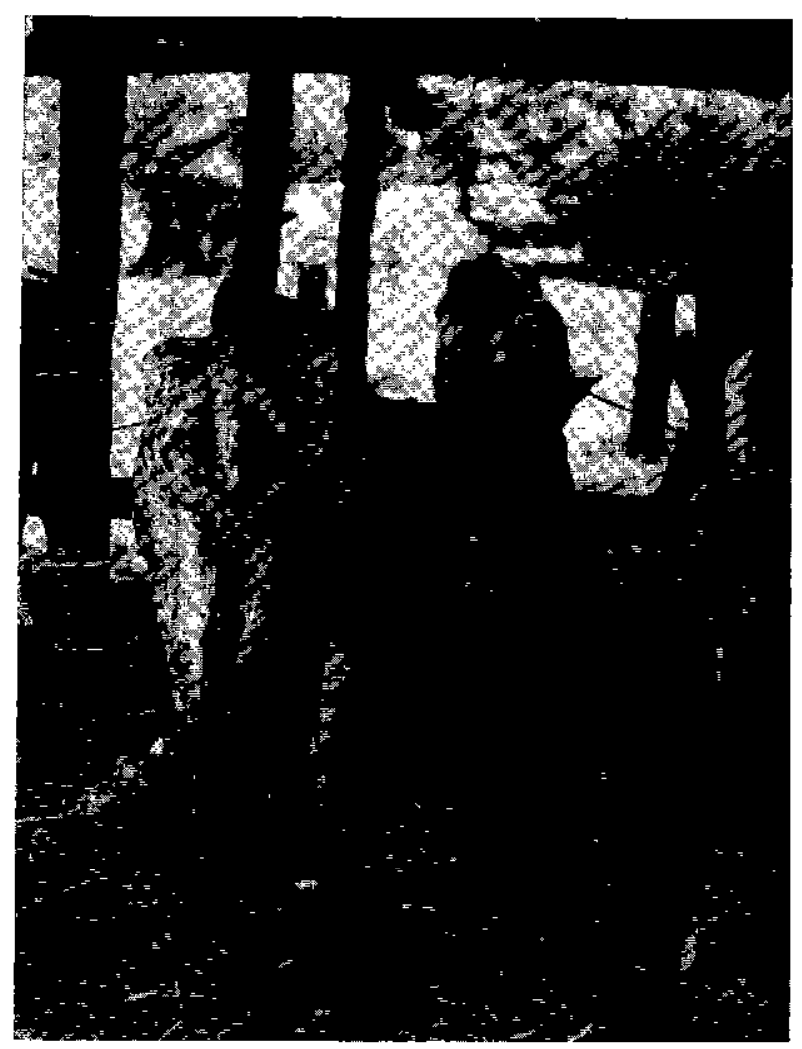

Photo $n^{0}$ 1. - Niger (juillet 1974).

Photo no 2. - Ethiopie (décembre 1976).

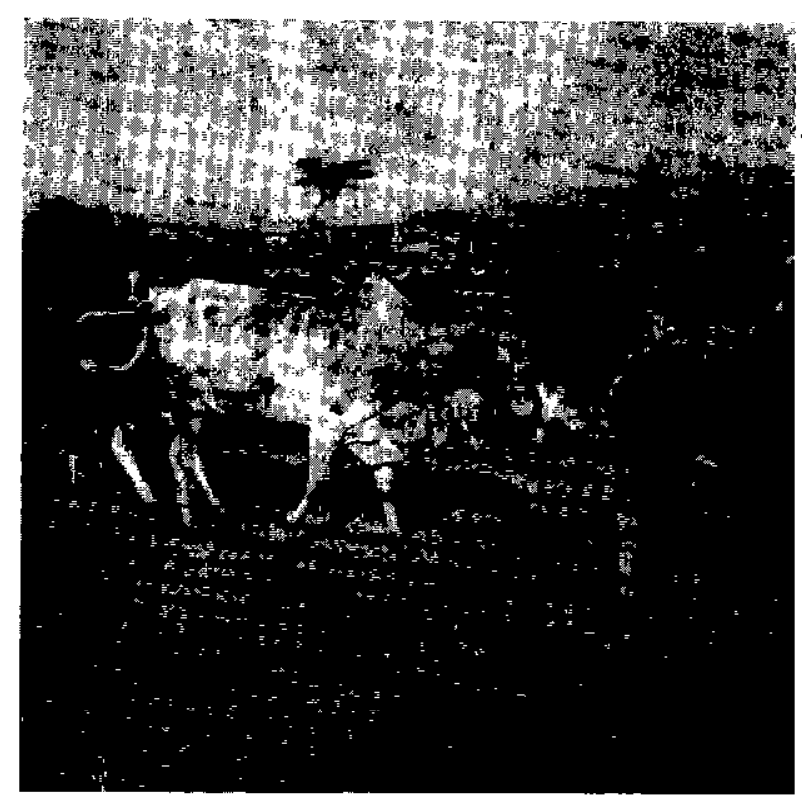


Ethiopie à $60 \mathrm{mg} / \mathrm{kg}$, il ne restait plus un seul animal porteur de paramphistomes adultes (Paramphistomum daubneyi). Seules, persistent les lésions du rumen sous forme d'érosions ou de destruction des papilles avec formation de grandes plages dénudées.

Sur un animal, il a été dénombré 25 Paramphistomum daubneyi immatures, ce qui est peu, le pourcentage global d'efficacité (adultes et immatures réunis) par rapport aux témoins étant supérieur à 99 p. 100.

Sur les paramphistomes juvéniles implantés dans le duodénum, on ne possède que peu de renseignements. Le Bitin-S à $35-50 \mathrm{mg} / \mathrm{kg}$ paraît détruire plus de 75 p. 100 des trématodes, comme le montrent des observations faites au Nigeria au cours d'une enzootie de paramphistomose ovine aiguë (21).

4. Au Niger, la tolérance des associations Disto-5 + Thiabendazole et Disto-5 + Morantel est excellente. Malgré l'état squelettique (Photo $\mathrm{n}^{0}$ 1) des animaux traités, aucun cas mortel n'a été observé.

L'association Tétramisole-Bitin-S est un peu moins bien supportée. Bien que n'ayant pas eu de mortalité à déplorer sur une période de 10 jours, on note, dans la moitié des cas, l'apparition d'une diarrhée, parfois violente, qui rétrocède assez rapidement. L'état général n'est pas perturbé pour autant et on ne constate aucune perte de poids.

On sait (4) que différents facteurs interviennent dans la bonne tolérance de cette association : sensibilité individuelle des animaux, intégrité du foie, régime alimentaire... Dans le cas de cette expérience, les animaux utilisés en Ethiopie étaient en assez bonne condition physique (Photo $n^{\circ}$ 2). Ils étaient "bourrés" de distomes hépatiques et d'échinocoques pulmonaires ou hépatiques. Du fait des pluies tardives, le pâturage était en pleine période de croissance avec des graminées courtes, vertes et très aqueuses. Aussi, dès le départ, les animaux présentaient-ils des fèces ramollies. L'administration de l'association Tétramisole-Bitin-S a aggravé temporairement cette situation, sans provoquer, cependant d'incidents dramatiques.

Dans d'autres pays, notamment en France, des associations de même type, mais de composition légèrement différente (Bithionol sulfoxyde $40 \cdot \mathrm{mg} / \mathrm{kg}+$ Tétramisole 10 à $14 \mathrm{mg} / \mathrm{kg}$ ) sont susceptibles d'occasionner chez les bovins des troubles divers nerveux, digestifs, respiratoires ou moteurs (8) imputables dans leur totalité à l'action du Tétramisole (4). La mort peut survenir par défaillance cardiaque ou au cours d'un épisode convulsif, 2 à 4 h plus tard.

D'une façon générale, dans le traitement des polyparasitoses, les associations médicamenteuses qui ne sont pas toujours inoffensives demandent à être choisies avec soin.

Les principes actifs entrant dans la composition de l'association, notamment le Tétramisole, doivent présenter toute garantie quant à leur origine, leur structure, leur pureté, leur innocuité et leur efficacité : c'est le cas du Tétramisole livré par les grandes firmes européennes (I. C. I., Rhône-Poulenc, Janssens...). Les accidents observés paraissent être le fait de lots de Tétramisole mal ou non contrôlés, de provenance douteuse ou inconnue, souvent achetés à l'étranger à bas prix.

Dans d'autres circonstances, c'est le mode d'administration qui est en cause : l'association sous forme de suspension se dépose, le Bithionol sulfoxyde vers le bas et le Tétramisole vers le haut. Si l'utilisateur n'agite pas suffisamment le bidon renfermant le médicament, le mélange se fait mal et la dose administrée aux premiers animaux traités comprend une quantité de Tétramisole 4 à 5 fois supérieure à la dose thérapeutique, ce qui explique en grande partie les troubles constatés.

Pour pallier cette difficulté, on a recommandé de donner séparément à quelques jours d'intervalle le Bitin-S et le Tétramisole. C'est aller à l'encontre du but recherché qui, outre l'élimination du plus grand nombre de parasites, vise à n intervenir qu'en une seule fois, en raison des problèmes que pose l'immobilisation d'animaux indociles dont la contention en Afrique, comme en Europe, est difficile.

Il vaut mieux modifier la présentation et utiliser lassociation :

- Soit sous forme de pâte à administrer par la voie buccale;

- Soit sous forme de poudre à mélanger à de la nourriture ;

- Soit sous forme de comprimés exactement dosés qui n'offrent pas les inconvénients de la suspension et qui, pour cette raison, doivent être recommandés en Afrique tropicale.

\section{CONCLUSIONS}

En Afrique tropicale où le polyparasitisme du zébu à base de «Strongles " gastro-intestinaux et de trématodes gastriques et hépatiques 
est fréquent, il est nécessaire, pour des opérations de déparasitage curatives ou préventives, d'utiliser des médicaments polyvalents à large spectre d'activité qui seront administrés selon un calendrier tenant compte des conditions épidémiologiques propres à chaque groupe d'helminthes.

En attendant la mise sur le marché de composés simples, on est bien obligé d'utiliser des associations de médicaments.

Des essais effectués au Niger et en Ethiopie ont montré que le Bithionol sulfoxyde à la dose de $40 \mathrm{mg} / \mathrm{kg}$ associé soit au Tétramisole $(10 \mathrm{mg} / \mathrm{kg})$, soit à du Thiabendazol ( 80 et $100 \mathrm{mg} /$ (10 $\mathrm{mg} / \mathrm{kg})$, soit à du Thiabendazole (80 et $100 \mathrm{mg} / \mathrm{kg}$ ), soit à du Tartrate de Morantel ( 5 et $7,5 \mathrm{mg} / \mathrm{kg}$ ) permettait de détruire la quasitotalité des formes adultes et mûres de Fasciola, de Paramphistomes, d'Haemonchus, de Cooperia et d'Oesophagostomum.

A l'autopsie, il reste:

- Dans la caillette, quelques Haemonchus avec le seul Tartrate de Morantel à $7,5 \mathrm{mg} / \mathrm{kg}$;

- Dans la panse, des Paramphistomes et des Carmyerius n'ayant pas encore atteint leur maturité sexuelle ;

- Dans le duodénum, des larves $\mathrm{L}_{4}$ inhibées de Cooperia punctata et de Coopera pectinata.

Compte tenu des observations faites au Niger, en Ethiopie, ainsi que dans d'autres pays d'Afrique (Nigeria), il importe de modifier légèrement la posologie des médicaments entrant dans la composition des associations, de manière à en accroître l'efficacité. La dose de Bithionol sulfoxyde devra être portée à $60 \mathrm{mg} / \mathrm{kg}$ : elle assure l'élimination à 99 p. 100 des paramphistomes adultes et immatures de la panse, ainsi que des Fasciola de plus de 6 semaines, ce qui rend plus sûres les interventions de la fin de la saison sèche, à une époque où ce type de parasitisme est dominant et économiquement grave.

De même, la dose de tartrate de Morantel sera augmentée $(10 \mathrm{mg} / \mathrm{kg})$. Pour le Thiabendazole et pour le Tétramisole, les doses expérimentées (100 et $10 \mathrm{mg} / \mathrm{kg}$ ) méritent d'être retenues.

La tolérance des associations Bithionol Sulfoxyde + Thiabendazole et Bithionol Sulfoxyde + Tartrate de Morantel est excellente.

L'association Bitın-S + Tétramisole est un peu moins bien supportée.

Pour les traitements de masse, les auteurs insistent sur la nécessité de n'utiliser en Afrique que des associations connues, sous marque déposée, fabriquées par des laboratoires capables de contrôler rigoureusement, donc de garantir la qualité de leur production et présentées sous forme de comprimés exactement dosés.

\section{REMERCIEMENTS}

Les auteurs tiennent à remercier vivement tous ceux qui ont facilité la réalisation de ces essais : le Gouvernement Nigérien, le Gouvernement Ethiopien, l'I. E. M. V. T., ainsi que les Sociétés Cogla et Pfizer international qui les ont financés.

\section{SUMMARY}

\section{Experimental treatment of polyparasitized zebu with combinations of Bitin-S + Thiabendazole, Bitin-S + Morantel Tartrate and Bitin-S + Tetramisole}

In tropical Africa, concurrent infections of zebu cattle with different species of parasites including gastro-intestinal roundworms and liver or stomach flukes are numerous and anthelmintic combinations have been used to extend the spectrum of activity in mixed parasitic infections.

Among them, combinations of Bithionol Sulfoxyde $(40 \mathrm{mg} / \mathrm{kg})+$ Thiabendazole ( 80 and $100 \mathrm{mg} / \mathrm{kg}$ ), Bithionol Sufoxyde $(40 \mathrm{mg} / \mathrm{kg})+$ Morantel tartrate $(5$ and $7,5 \mathrm{mg} / \mathrm{kg})$, Bithionol Sulfoxyde $(40 \mathrm{mg} / \mathrm{kg})-T$ Tetramisole $(10 \mathrm{mg} / \mathrm{kg}$ ) were shown, in Niger and Ethiopia, to be effective on Fasciola (more than eight weeks old), adult Paramphistomes, Haemonchus, Cooperia and Oesophagostomum.

However, to provide better activity against certain Nematodes (Haemonchus), Fasciola more than six weeks old and young Paramphistomum, the dose of Bithionol Sulfoxyde must be slightly increased $(60 \mathrm{mg} / \mathrm{kg}$ ). Morantel tartrate should be changed from 7 to $10 \mathrm{mg} / \mathrm{kg}$, while Thiabendazole and Tetramisole remain inchanged at 100 and $10 \mathrm{mg} / \mathrm{kg}$.

A little diarrhoea was observed with Bithionol Sulfoxyde + Tetramisole combination, but no side effects with two others.

The authors emphasize the point to use only registered trade-mark combunations guaranteed by known manufacturers and administered in exactly dosed pellets. 


\title{
RESUMEN
}

\begin{abstract}
Ensayo de tratamiento, en las condiciones africanas, del poliparasitismo del cebú mediante asociaciones Disto-5-thiabendazole, Disto-5-Tartrato de Morantel y Disto-5-Tetramisole
\end{abstract}

En Africa tropical, se encuentra frecuentemente el poliparasitismo del cebú a base de Nemátodos y de Tremátodos y es necesario, para luchar contra él, de utilizar los antıhelminticos o las asociaciones de medicamentos teniendo un amplio espectro de actividad.

Entre estas, asociaciones incluyendo Bithionol sulfoxido $(40 \mathrm{mg} / \mathrm{kg})+$ Thiabendazole $(80$ y $100 \mathrm{mg} / \mathrm{kg})$, Bithionol sulfoxido $(40 \mathrm{mg} / \mathrm{kg})+$ Tartrato de Morantel (5 y $7,5 \mathrm{mg} / \mathrm{kg}$ ) Bithionol sulfoxido $(40 \mathrm{mg} / \mathrm{kg}$ ) + Tetramisole $(10 \mathrm{mg} / \mathrm{kg}$ ) se mostraron particularmente eficaces para con Fasciola de más de ocho semanas, Paramfistomos de la panza, Haemonchus, Esofagostomos y Cooperia adultos, durante ensayos efectuados en el Niger y en Etiopia.

Sin embargo, para aumentar su eficacia para con ciertos Nemátodos (Haemonchus), Fasciolas de menos de ocho semanas de edad y Paramfistomos maduros o inmaduros, se debe elevar la dosis hasta $60 \mathrm{mg} / \mathrm{kg}$ y la del Morantel hasta $10 \mathrm{mg} / \mathrm{kg}$, las dosis de Thuabendazole y de Tetramisole quedando sin cambiar ( 100 y $10 \mathrm{mg} / \mathrm{kg}$ ).

La asociación Bithıonol-Sulfoxido - Tetramisole parece un poco menos bien tolerada que las dos otras.

Los autores insisten en la necesıdad, en Africa, de escoger asociaciones bajo marca registrada fabricadas por laboratorios capaces de garantizar rigurosamente su producción y presentadas bajo forma de tabletas exactamente dosificadas.

\section{BIBLIOGRAPHIE}

1. BELL (R. R.), GALVIN (T. G.), TURK (R D.). Anthelmintics for ruminants. VI. Thiabendazole. Am. J. vet. Res., 1962, $93:$ 195-199.

2. BOUCHET (A.), GRABER (M.). Etude de l'action anthelminthique du Tétramisole vis-à-vis des principaux nématodes du veau de lait. Essais réalisés en milieu tropical humide. Cah. Méd. vét., 1969, 38 (5) : 156-164.

3. CIORDA (H.), McCAMPBELL (H. C.). Anthelmintic activity of Morantel tartrate in calves. $\mathrm{Am}$. J. vet. Res., 1973, $34(5): 619-620$

4. DELATOUR (P.), GRABER (M.). Toxicité chez les ruminants de l'association Tétramisole-Bithıonol Sulfoxyde. Lyon, Centre natn. Inf. Toxicol. vet., 1977 (I) : 15-17.

5. DEMIDOV (N. V.), RAINHER (Sh. G.). The efficacy of Sulphene against Fasciola in cattle. Byull. vses. Inst. gel' mint K. I. Skryabina, 1971 (5) : 21-23 (en russe).

6. EUZEBY (J.). Diagnostıc expérımental des helminthoses animales. Paris, Vigot frères, 1958.

7. GRABER (M.). Etude en milieu tropical de l'action antiparasitaire du Thiabendazole sur divers helminthes des animaux domestiques. I. - Helminthes du zébu. III. - Nématodes du veau de lait. Rev. Elev. Méd. vét. Pays trop., 1965, 18 (1) : 39-58 et 1968, 21 (1) : 71-78

8. GRABER (M.). Etude du pouvoir anthelmınthıque et de la toxicité d'un nouveau médicament, le Tétramisole (16.535 R. P.) chez le mouton et chez le zébu des zones tropicales. Cah. Méd vét., 1967, 36 (2) : 1-23.

9. GRABER (M.). Les Trématodoses hépatiques et gastriques des zébus d'Afrique centrale. Rev. Elev. Méd. vét. Pays trop., 1975, 28 (3) : 311-314.

10. GRABER (M.), BIRGI (E.), TRONCY (P. M.). A propos de l'action du Bitin-S et du Bromophénophos sur les formes immatures de Fasciola gigantica. Rev. Elev. Méd. vét. Pays trop., 1971, 24 (1) : 37-41.

11. GRABER (M.), DELAVENAY (R), GEBRE NEGUS TESFAMARIAN. - Inventaire parasitolo- gique de l'Ethiopie. Helminthes des zébus adultes de la région de Kofélé (Arussi, Ethiopie). Rev. Elev. Méd. lér. Pays trop., 1978 : 31 (3) : 341-352

12. GRABER (M.), TAGER-KAGAN (P.). Inhibition du développement des larves de Cooperia punctara et de Cooperia pectinata chez le zébu nigérien. Rev. Elev. Méd. vét. Pays trop., 1975, 28 (2) : 137-142.

13. GUILHON (J.), GRABER (M.). Etude du pouvoir authelminthique du bis (2-hydroxy-3,5-dichlorophényl) Sulfoxyde en milieu tropical. Rev. Elev. Méd. vét. Pays trop., 1967, 20 (1): 87-104.

14. GUILHON (J.), GRABER (M.). Action d'un dérivé chloré de la Salicylanilide en milieu tropical sur les Trématodes parasites des bovins. Rev. Elev. Méd. vét. Pays trop., 1971, 24 (3) : 365-371.

15. HERLICH (H.). Efficacy of Thiabendazole as an anthelmintic in cattle and sheep. Vet. Med., 1963, 58 (11) , 874-882.

16. HORAK (I. G.), SNIJDERS (A. J.), PIENAAR (I. T.). The efficacy of Cambendazole against Cestodes and Nematodes infestations in sheep and cattle. Il. S. Afr. vet. Ass., 1972, 43 (1) : 101-106.

17 RAIKHER (Sh. G.), KONTRAL'EV (V. P.), KAS' YANOV (F. I.), LYSENKO (N. M.). Trials of Sulphene and KP-5 against Fasciolıasis in rumınants (Part II) Veterinariya, Moscou, 1975, 52 (9) : 74-75 (en russe).

18. Rapport annuel de la région de recherches vétérinaires et zootechniques d'Afrique centrale, Laboratoire Vétérinaire de Farcha (Tchad), 1969, 1 : 273-274.

19. RAYNAUD (J P.). Un anthelminthique actif sur les Strongles digestifs des petits ruminants, le Tartrate de Morantel. Rec. Méd. vét., 1972, 148 (5) . 591-602.

20. SCHILLHORN VAN VEEN (T.). The efficacy of four antheimintics in adult cattle in field conditions (à paraître).

21. SCHILLHORN VAN VEEN (T.), BIDA (S. A.). Acute paramphistomiasis in sheep in Zaria. Proc. 20th Wld. vet. Cong., Thessaloniki, 1975, 1 : 517-519. 
22. SEY (O.), GRABER (M.). Examination of Amphistomes (Trematoda : Paramphistomidae) of some african mammals. Rev. Elev. Méd. vét. pays trop. (à paraître).

23. THEODORIDES (V. J.), GYURIK (R. J.), KINGSBURY (W. D.), PARISH (R. C.). Anthelmintic activity of Albendazole against liver flukes, tapeworms, lung and gastro-intestinal roundworms. Experientia, 1976, 32 (6) : 702-703.
24. TRONCY (P. M.), OUMATIE (O.). Emploi du Tartrate de Morantel chez le zébu du Tchad. I. Action sur les Strongylidae. II. - Action sur les Nématodes du veau de lait. Rev. Elev. Méd. vét. Pays trop., 1973, 26 (2) : 188-198 et 199-202.

25. TRONCY (P. M.), VASSEAU-MARTIN (N.). Le Rafoxanide dans le traitement de la fasciolose à Fasciola gigantica chez le zébu du Tchad. Rev. Elev. Méd. vét. Pays trop., 1976, 29 (1) : 31-7. 